\title{
Proposta de RESPOSTA A PARTIR DA COMPARAÇÃO ENTRE "ENVIRONMENT AND BEHAVIOR" E "JOURNAL OF ENVIRONMENTAL PSYCHOLOGY"
}

\author{
Maria Vittoria Giuliani ${ }^{1}$ \\ Universidade de Roma "La Sapienza"
}

\begin{abstract}
Este artigo define o campo em Psicologia Ambiental a partir de dados referentes aos periódicos Environment and Behavior $e$ Journal of Environmental Psychology. Para isto, baseia-se na tipologia de Stokols que caracteriza a transação humano-ambiente em duas dimensões básicas: formas de transação cognitiva (ou simbólica) x comportamental (ou física); e fases de transação ativa x reativa, gerando quatro modos de transação: interpretativa; avaliativa; responsiva; operativa. Conclui que diferentes modos de transação pessoas-ambiente são tanto áreas estáveis de interesse quanto há diferenças entre os periódicos. Conclui que a importância do contexto sociocultural sobre o objeto de estudo estaria implícita na própria definição de ambiente. Ao final, estabelece a diferença entre pesquisa aplicada e orientada.
\end{abstract}

Descritores: Psicologia ambiental. Objeto. Fatores socioculturais. Pesquisa. Periódicos.

\begin{abstract}
A credito que um curso introdutório de qualquer disciplina começa com uma definição do campo: "O que é ***? Com o que ela lida? etc.", mas gostaria de saber quantos setores científicos existem nos quais a questão
\end{abstract}

1 Pesquisadora Senior do Instituto de Ciências Cognitivas e Tecnologias do Conselho Nacional de Pesquisa (CNR-Itália), especialista em Psicologia Ambiental e coordenadora de pesquisas na Universidade de Roma "La Sapienza". Endereço eletrônico: vittoria.giuliani@istc.cnr.it 
parece problemática mesmo para aqueles que trabalham no campo, como é o caso da Psicologia Ambiental. Porque a dificuldade em definir o campo?

Alguns anos atrás, um artigo de Stokols (1995), que apareceu na American Psychologist, disparou um debate sobre a identidade da Psicologia Ambiental. Nele, o autor sustentava que a identidade do setor como uma área específica de estudo - isto é, como um campo caracterizado por um paradigma específico - tinha se tornado crescentemente "difusa" e "transparente" por três razões fundamentais: a sua complexidade multidisciplinar, o seu escopo internacional, e a incorporação de questões ambientais em todos ou quase todos os setores da psicologia. De acordo com Stokols, a Psicologia Ambiental tenderia a continuar perdendo a sua identidade porque cada vez mais os seus princípios conceituais e metodológicos estão sendo incluídos em outras áreas da psicologia e em outras disciplinas como um resultado de uma crescente conscientização da necessidade de uma abordagem "contextual" para resolver problemas sociais importantes, como poluição e a qualidade total do ambiente, violência urbana, o impacto de novas tecnologias sobre o trabalho e a vida cotidiana, na saúde, e o envelhecimento da população mundial.

Mais recentemente, Sime (1999), a partir de uma pesquisa em seis manuais sobre Psicologia Ambiental, publicados entre 1995 e 1997, intitulado - não coincidentemente - "O que é a Psicologia Ambiental", mostra como as visões de vários estudiosos diferem tanto quanto ao paradigma teórico e às metodologias que definem este campo de pesquisa, quanto ao papel assinalado para a psicologia como parte do estudo das relações entre as pessoas e o ambiente.

Finalmente, Bonnes e Bonaiuto (2002) avaliam que "nos últimos 15 anos, por meio do aumento da influência da perspectiva ecológica plena, o ambiente ecologicamente considerado se tornou crescentemente central para a Psicologia Ambiental", com a Psicologia Ambiental sendo crescentemente vista como "psicologia ambiental da sustentabilidade".

Contudo, muitas das diferenças em definir a Psicologia Ambiental surgem não da análise do que está sendo estudado e de como aqueles que se 
definem como psicólogos ambientais a estudam, mas das diversas avaliações do que deveria ser feito: "é" usualmente significa "deveria ser".

Portanto, penso ser útil tentar responder à questão em termos empíricos / operacionais, supondo que o objeto da Psicologia Ambiental é com o que os psicólogos ambientais lidam ou, para ser mais precisa, sobre o que estão escrevendo.

Com este propósito, escolhi selecionar as duas publicações internacionais mais representativas no campo para ver se e como os interesses daqueles que se identificam com o nome de Psicologia Ambiental foram orientados nos últimos anos. Tenho consciência de que uma grande parte da atividade realizada neste campo não está refletida nestes periódicos - em particular, a pesquisa aplicada está definitivamente sub-representada (o quadro poderia ser diferente se fôssemos examinar as contribuições apresentadas na IAPS 2 e EDRA ${ }^{3}$ ), e que o mundo dos de fala inglesa está sobrerepresentado com respeito às contribuições da comunidade que não fala inglês - contudo, os dois periódicos, em seus muitos anos de publicação certamente oferecem um quadro bastante completo da disciplina.

Aqui temos os dados essenciais concernentes aos dois periódicos Environment and Behavior (E\&B) e Journal of Environmental Psychology (JEP). Os programas editoriais incluem uma definição geral do campo e alguns indicadores sobre possíveis tópicos, como veremos a seguir.

\section{Environment and Behavior}

1. Foi fundada em 1969 (com apenas 2 números naquele ano), tendo sido uma publicação trimestral de 1970 a 1980; e 6 números por ano, desde 1981. O último número examinado foi o vol. 34 , n. 5 e o total de artigos publicados até o momento é 1025 .

2 IAPS: International Association for People-Environment Studies.

3 EDRA: Environment Design Research Association. 


\section{Programa editorial:}

- trabalhos teóricos sobre as inter-relações entre ambientes humanos e sistemas comportamentais e trabalhos metodológicos quando o foco primário se refere à relação ambiente-comportamento;

- relatos de pesquisa relacionados com a avaliação de ambientes planejados para cumprir com objetivos específicos, por ex., os efeitos sociais de diferentes tipos de alojamento ou a efetividade de áreas de tratamento hospitalar;

- estudos relacionados a crenças, significados, valores e atitudes de indivíduos ou grupos referentes a vários ambientes, por ex., o significado e os valores ligados à vizinhança, cidades, vias e dispositivos de transporte, ou área de lazer;

- estudos referentes a ambientes físicos onde a missão humana está amplamente implícita ou socialmente subdesenvolvida;

- estudos sobre o planejamento, política, ou ação política objetivando controlar ambientes ou comportamentos.

\section{Journal of Environmental Psychology}

1. Foi fundado em 1981, é publicado trimestralmente, o último número examinado foi o vol. 22, n. 3 e o total de artigos publicados até o momento é 509.

2. Programa editorial:

- artigos empíricos e conceituais que possibilitam o avanço da compreensão das relações entre as pessoas e o seu entorno físico. São contemplados os aspectos teóricos, metodológicos e práticos dessa compreensão procurando promover o desenvolvimento científico deste campo interdisciplinar.

- o entorno físico inclui tanto o ambiente construído e feito pelo homem quanto o ambiente natural.

- o objetivo do JEP é publicar artigos de alto padrão acadêmico que têm o potencial de aumentar o bem-estar humano. 
Selecionar os critérios para dassificar os muitos artigos publicados desde então (o número total de artigos soma 1534) não é uma tarefa fácil; basta ver os levantamentos sobre o JEP publicados pelo Annual Review of Psychology para verificar quantos diferentes critérios podem ser adotados.

Escolhi três critérios de classificação:

O primeiro é o conjunto estabelecido por Stokols (1978) que considera os vários modos de transação humano-ambiente; o segundo é o tipo de ambiente considerado; e o terceiro é a tipologia humana. Nesta apresentação, tratarei apenas do primeiro.

Segundo Stokols (1978), a transação humano-ambiente "pode ser caracterizada em termos de duas dimensões básicas: 1. Formas de transação cognitiva (ou simbólica) x comportamental (ou física); e 2. Fases de transação ativa $x$ reativa. Consideradas conjuntamente, essas dimensões produzem quatro modos de transação humano-ambiente, como mostrado no Quadro 1.

Quadro 1. Os quatro modos de transação humano-ambiente (citado por Stokols, 1978)

\begin{tabular}{|c|c|c|c|}
\hline \multirow{4}{*}{$\begin{array}{l}\text { FASE DE } \\
\text { TRANSAÇÃO }\end{array}$} & \multirow{4}{*}{$\begin{array}{l}\text { Ativa } \\
\text { Reativa }\end{array}$} & \multicolumn{2}{|c|}{ FORMA DE TRANSAÇÃO } \\
\hline & & Cognitiva & Comportamental \\
\hline & & Interpretativa & Operativa \\
\hline & & Avaliativa & Responsiva \\
\hline
\end{tabular}

Excluí da análise os trabalhos que tratavam de questões gerais teóricas, metodoló gicas ou de planejamento, que não se ajustavam ao esquema acima $(\mathrm{n}=228)$. As maiores áreas de pesquisa foram caracterizadas segundo o modo pelo qual foram tipicamente focalizadas, obtendo-se o seguinte esquema:

1. modo interpretativo: cognição espacial, mapas cognitivos, labirintos, percepção do ambiente; significado e sentido de lugar, lugar e processos de identidade, processos afetivos; personalidade e o ambiente; 
2. modo avaliativo: avaliação de qualidade ambiental, preferências e satisfação; atitudes ambientais e disposição, preocupação ambiental e valores, percepção de risco;

3. modo operativo: comportamento pró-ambiental (reciclagem, lixo, uso de energia etc.), intervenções para preservar o ambiente; controle do ambiente, territorialidade e privacidade, espaço pessoal; organização e uso do espaço, escolhas locacionais, participação em planejamento;

4. modo responsivo: conseqüências comportamentais e de saúde de estressores, comportamento de enfrentamento; impacto do ambiente construído/natural sobre desempenho, saúde e comportamento.

Ás vezes, algumas simplificações foram necessárias dado que, como Stokols (1978) observou, os limites entre os diferentes modos não são sempre claros e distintos, e numerosos estudos compreendem mais do que um único modo. Por exemplo, os estudos sobre densidade ou apinhamento freqüentemente examinam não apenas as reações das pessoas mas também aspectos de avaliação e interpretação; atitudes pró-ambientais são freqüentemente consideradas em relação ao comportamento, etc. Além do mais, a classificação é ainda provisória e planejo realizar uma avaliação posteriormente. Contudo, mesmo com essas limitações, um quadro geral do campo pode ser derivado.

A comparação de um espaço relativo devotado aos diferentes modos (ver Tabela 1), durante a vida inteira dos periódicos, mostra diferenças significativas $(\chi 2=51,788, \mathrm{df}=3, \mathrm{p}<.001)$.

Em ambos, e mais no JEP, a forma de transação a quem foi dada prioridade é o modo cognitivo. Em particular, o modo interpretativo parece ocupar um terço dos artigos do JEP, enquanto a maior ênfase do J\&B está no modo avaliativo. 
Tabela 1: Porcentagem de artigos focalizando cada modo transacional

\begin{tabular}{|l|c|c|c|}
\hline Modo transacional & E\&B $(\%)$ & JEP $(\%)$ & Total \\
\hline Interpretativo & 16,38 & 33,79 & 22,23 \\
\hline Avaliativo & 34,92 & 31,96 & 33,92 \\
\hline Operativo & 23,32 & 15,98 & 20,85 \\
\hline Responsivo & 25,41 & 18,26 & 23,00 \\
\hline Total & 100 & 100 & 100 \\
\hline
\end{tabular}

Mais diferenças podem ser notadas quando as tendências da publicação em cada área tópica são comparadas. $O$ interesse no estudo da representação cognitiva do ambiente parece ser uma característica estável de JEP pelos anos, enquanto parece ter havido um decréscimo em E\&B nos anos 90, acompanhado por um ligeiro aumento em artigos centrados em significado ambiental, afeto e identidade. Em ambas publicações, o número de artigos devotados à personalidade e ambiente é desprezível.

Quanto ao modo avaliativo, em ambos os periódicos, a distribuição das duas áreas de avaliação ambiental e de atitudes ambie ntais parece se alternar: à medida que uma cresce, a outra decresce. No E\&B, os artigos sobre atitudes ambientais e interesse estavam entre os mais numerosos na primeira década (1970s), então diminuíram nos anos de 1980 para aumentar em número de novo e tornar-se a área com mais artigos na segunda metade dos anos de 1990. Em JEP, o tópico recebeu uma atenção maior somente nos últimos dois anos.

Há também diferenças significativas entre os dois periódicos com referência aos modos operativo e responsivo, ambos sendo significativamente mais presentes em E\&B do que em JEP. 
É interessante notar que a distribuição de estudos sobre o comportamento pró-ambiental em $\mathrm{E} \& \mathrm{~B}$ mostra uma tendência similar àquela das atitudes ambientais, em particular referente ao seu forte crescimento na segunda metade dos anos 1990. Disse anteriormente que os limites entre estudos sobre atitudes e comportamentos pró-ambientais são arbitrários, dado que muitos estudos focalizam a relação entre os dois. $\mathrm{O}$ recente aumento em contribuições em ambas as áreas faz pensar que o elemento decisivo é o interesse pelo ambiente, antes do que por um ou outro modo transacional. De outro lado, os estudos sobre controle ambiental, privacidade e territorialidade parecem estar decrescendo, enquanto a área que engloba os estudos sobre o uso e mudanças no ambiente parece estar estável.

Quanto ao JEP, a despeito de um maior interesse no tema comportamento pró-ambiental na segunda metade dos anos 1990, isto não foi confirmado nos últimos dois anos.

Finalmente, o interesse no modo responsivo parece ser estável através dos anos, mesmo se parece haver menos interesse em ambos os periódicos sobre o estudo do impacto de aspectos específicos do ambiente natural ou construído sobre o desempenho, o bem-estar psicológico e a interação social. Ao mesmo tempo, a tendência em estudar as reações aos estressores ambientais pode ser menos claramente definida.

Em geral, podemos dizer que os diferentes modos de transação pessoas-ambiente são todas áreas estáveis de interesse para a Psicologia Ambiental. Mas a comparação dos dois periódicos também ajuda a apontar certas diferenças entre a Psicologia Ambiental no sentido estrito e o estudo das relações pessoas-ambiente em sentido amplo. Mesmo se ambos os periódicos consideram o último uma área interdisciplinar, JEP parece atribuir à psicologia um lugar bem mais central do que $\mathrm{E} \& \mathrm{~B}$, e isto se reflete na maior ênfase dada à interpretação do ambiente. De modo contrário, o maior interesse nos modos reativos de transação, seja avaliativa ou responsiva, parece refletir um maior envolvimento do componente projeto e planejamento.

Se for verdade que a diferença na ênfase dada pelos dois periódicos aos vários modos de transação entre as pessoas e o ambiente depende da 
diferença nos componentes disciplinares, isto pode proporcionar um indicador para responder à questão de como o contexto sociocultural pode levar a diferentes visões de Psicologia Ambiental.

A importância do contexto sociocultural sobre o objeto de estudo está de fato implícita na própria definição de ambiente como um ambiente real, não apenas físico mas sócio-físico.

Em cada país, as condições de vida de algumas partes da população parecem problemáticas; no entanto, lidar com as favelas do Rio ou de São Paulo ou com moradias de baixa renda em um bairro de imigrantes em Estocolmo, certamente demanda o desenvolvimento de instrumentos conceituais e metodológicos diferentes.

A esse respeito, deixem-me contar uma história curta e divertida. $\mathrm{O}$ primeiro congresso sobre Psicologia Ambiental que freqüentei tinha algumas sessões devotadas à pesquisa sobre moradia e qualidade residencial. Não me recordo muito bem dos trabalhos apresentados - foi o 20th International Congress of Applied Psychology, realizado em Edimburgo em 1982 mas ainda tenho uma lembrança bem vívida de uma intervenção de uma pessoa que assistia à sessão. Era um colega da Iugoslávia que comentou que a questão de medir a qualidade do ambiente residencial era um tópico fascinante, e que ele gostaria de ter feito alguma pesquisa sobre isto mas que, infelizmente, em seu país era muito difícil: lá, a insuficiência quanto a moradias era tão grande que qualquer tentativa de avaliar a importância relativa de moradias diferentes e de características de vizinhança sobre a satisfação das pessoas seria impossível. De fato, as pessoas ficavam tão felizes, depois de longos anos de espera, de ter qualquer moradia, que a sua qualidade era totalmente irrelevante.

Uma análise mais específica de ambas diferenças - as diferenças em importância dada às questões estudadas nos últimos 30 anos e as diferenças entre países -, demandaria tempo e competências que não possuo. Contudo, algumas indicações, mesmo que agora ultrapassadas, podem ser obtidas por meio de uma comparação dos vários capítulos do Handbook of Environmental Psychology, de 1987, sobre "Perspectivas Internacionais em Psicologia 
Ambiental" e os artigos de vários países que apareceram em JEP e E\&B em 1981/82. Anos atrás, Pol (1988) fez um excelente trabalho deste tipo sobre a Europa no livro La Psicología Ambiental em Europa. Eu apenas apontaria que as diferenças são provavelmente maiores do que aparentam a partir destes textos porque a literatura internacional é dominada pela América do Norte.

Contudo, percebo que pode haver um outro significado, talvez menos óbvio, por detrás das relações entre a realidade sociocultural e a Psicologia Ambiental, qual seja, a de que a diversidade determinada pela peculiaridade sociocultural não se refere tanto ao objeto de estudo quanto ao contexto de produção, ou em outras palavras, à organização social da pesquisa. Peço desculpas se estiver invadindo, de algum modo, o território das próximas sessões, mas percebo que há dois elementos substanciais que caracterizam as diferentes visões de Psicologia Ambiental. Eles são o grau da ênfase colocada na pluri-disciplinaridade ou a natureza interdisciplinar deste setor de estudo, e a ênfase colocada nos aspectos teóricos da Psicologia Ambiental em relação aos aspectos aplicados.

Quanto ao primeiro, gostaria de retornar ao artigo de Sime (1999) mencionado anteriormente. Nessa análise, um amplo espaço é dedicado à discussão dos diferentes modos pelos quais a relação entre a Psicologia Ambiental e as várias disciplinas relacionadas ao ambiente - a psicologia e outras ciências do comportamento e sociais, ciências do planejamento e físicas - apresenta-se nos seis textos revistos. A Psicologia Ambiental é vista em um extremo de uma sub-área da psicologia social e, de outro lado, como uma área inter-disciplinar de estudo, talvez mais adequadamente chamada de "estudos ambiente e comportamento", que tem recebido contribuições de diversos setores disciplinares, mas é autônoma com respeito a cada um deles.

Sime não propõe nenhuma hipótese quanto ao motivo para essas diferenças, mas suspeito fortemente que elas têm menos a ver com as preferências pessoais dos autores do que com o grau de rigidez da cultura acadêmica na qual eles operam e da possibilidade de conflitos ou alianças disciplinares que prejudicam ou favorecem a colaboração entre os diferentes setores. Seria interessante explorar se a estrutura acadêmica está ligada, não apenas a 
uma definição diferente do setor, mas também ao modo pelo qual o setor se desenvolve. Por exemplo, o fato de a Psicologia Ambiental ser menos desenvolvida e ter se desenvolvido posteriormente na Europa continental, em relação à Grã-Bretanha e à América do Norte, poderia ser um decorrência de uma menor flexibilidade da cultura acadêmica européia?

As diferenças mencionadas acima se referem ao "o quê" e ao "como" da Psicologia Ambiental, mas pouco dizem do "porquê". Tem sido dito que a pesquisa em Psicologia Ambiental é "centrada-no-problema" antes do que "centrada-na-teoria" (Darley \& Gilbert, 1985); em outras palavras, o propósito da pesquisa em Psicologia Ambiental não é a mera observação, descrição e explicação do modo pelo qual os seres humanos interpretam o ambie nte, operam nele e reagem a ele, mas uma procura por instrumentos adequados para promover uma mudança que melhore o bem-estar humano. $\mathrm{E}$, deve ser destacado que falar de mudança nas relações humano-ambiente significa que a mesma importância é dada à mudança do ambiente físico como para mudar os modos pelos quais as pessoas interferem no ambiente (pense, por exemplo, sobre a participação na tomada-de-decisão e no desenvolvimento de políticas públicas pró-ambientais), ou quanto a mudar as atitudes ou conceitos das pessoas sobre qualidade ambiental (por exemplo, a transformação implicada nas expressões "projetado para todos" com respeito a "planejado para pessoas portadoras de deficiências" ou "para idosos").

Quanto à centralidade da orientação multidisciplinar na definição do setor, a visão dos estudiosos também diverge quanto à centralidade da orie ntação para a mudança. Depende apenas das inclinações pessoais, como argumenta, melhor do que eu, Esther Wiesenfeld, das suposições filosóficas que guiam a pesquisa, ou das diferenças originadas do contexto sociocultural?

Gostaria de frisar as profundas diferenças que percebo entre os termos "pesquisa aplicada" ou "pesquisa orientada-para-a-mudança" ou simplesmente "pesquisa orientada". Por pesquisa aplicada quero dizer aquela que responde a uma solicitação de uma solução a um problema, usando conhecimento e técnicas formuladas em um ou mais campos disciplinares e adaptando-as ao problema particular. A pesquisa orientada, por outro lado, é a- 
quela que contribui para a identificação dos problemas formulando os conceitos e os constructos interpretativos que modificam a nossa visão do mundo ou, no nosso caso, as relações humano-ambientais. Portanto, a pesquisa orientada é mais proposicional do que instrumental. Para dar um exemplo concreto, estamos fazendo pesquisa aplicada quando usamos as nossas competências sobre satisfação residencial para formular recomendações quanto às relações entre parques e construções ou quanto à altura das edificações no planejamento urbano. Estamos fazendo pesquisa orientada, contudo, quando reformulamos o problema da satisfação residencial em termos não apenas das características do contexto físico mas, por exemplo, do significado que ela assume para os moradores, como foi realizado nas formulações dos conceitos de identidade de lugar ou de apego ao lugar.

Cerca de dez anos atrás, Jacques Curie (1990) iniciou uma conferência dedicada à relação entre psicologia e sociedade dizendo "a demanda social é cega sem a pesquisa e a pesquisa debilita-se sem a primeira". Isto é mais verdade ainda quando os objetivos da pesquisa - como em Psicologia Ambiental - incluem mudar as relações entre as pessoas e o ambiente, um ambiente que não é nunca apenas físico, mas sócio-físico, e quando mudar freqüentemente implica em mudar o equilíbrio de poder entre os vários atores sociais. Porém, a fim de que a pesquisa e a sociedade em geral interajam proveitosamente, deve haver interesse e capacidade, por parte da sociedade, de fazer com que a pesquisa formule modelos interpretativos e não somente proporcione soluções específicas. A esfera da pesquisa, por outro lado, tem de ter o interesse e a capacidade de reconhecer os problemas importantes e fazer o esforço criativo de descobrir os seus aspectos menos evidentes.

Acredito que apenas nestas condições pode a natureza orientada-aoproblema da Psicologia Ambiental ser amplamente compartilhada e não permanecer em uma aspiração mais ou menos ilusória de indivíduos. 
Giuliani, M. V. (2005). Proposal for an answer based on the comparison between "Environment and Behavior" and "Journal of Environmental Psychology”. Psicologia USP, 16(1/2), 89-102.

\begin{abstract}
This article defines the field in Environmental Psychology based on data referring to the journals Environment and Behavior and Journal of Environmental Psychology. To accomplish this, it uses Stokols' typology, which characterizes the human-environment transaction in two basic dimensions: cognitive (or symbolic) x behavioral (or physical) transaction forms; and active $\mathrm{x}$ reactive transaction phases, generating four transaction modes: interpretative; evaluative; responsive; operative. It concludes that different people-environment transaction modes are stable areas of interest; however, there are differences between the journals. The importance of the socio-cultural context on the object of study would be, therefore, implicit in the definition of environment. Finally, it establishes the difference between applied and oriented research.
\end{abstract}

Index terms: Environmental psychology. Object. Sociocultural factors. Research. Journals.

Giuliani, M. V. (2005). Proposition de réponse a partir de la comparaison entre "Environment and Behavior" et "Journal of Environmental Psycholog”. Psicologia USP, 16(1/2), 89-102.

Résumé: Cet article définit le domaine dans la Psychologie de l'Environnement à partir de données relatives aux périodiques Environment and Behavior et Journal of Environmental Psychology. Pour ce faire, il se fonde sur la typologie de Stokols qui caractérise la transaction humaineenvironnementale en deux dimensions de base: formes de transaction cognitive (ou symbolique) x comportemental (ou physique); et phases de transaction active $\mathrm{x}$ réactive, produisant quatre modes de transaction : l'interprétative; l'estimative; la sensible; l'opérative. Il en conclut que différents modes de transaction personnes-environnement représentent autant de points stables d'intérêt qu'il y a de différences entre les périodiques. I'importance du contexte socioculturel concernant l'objet d'étude serait, de cette façon, implicite dans la propre définition d'environnement. Finalement, il établit la différence entre recherche appliquée et recherche orientée.

Mots-clés: Psychologie de l'environnement. Objet. Aspects socioculturels. Recherche. Périodiques. 


\section{Maria Vittoria Giuliani}

\section{Referências}

Bonnes, M., \& Bonaiuto, M. (2002). Environmental psychology: From spatial-physical environment to sustainable development. In R. B. Betchel \& A. Churchman (Eds.), Handbook of environmental psychology (pp. 28-54). New York: John Wiley \& Sons.

Curie, J. (1990). Enjeux et défis de l'articulation des réseaux scientifiques et de la demande sociale en psychologie. In Laboratoire "Personnalisation et changements sociaux" (Ed.), Actes du Colloque International sur La recherche en psychologie en Europe. Demandes sociales et reseaux scientifiques (pp. 2-6). Toulouse: Université de Toulouse Le Mirail.

Darley, J. M., \& Gilbert, D. T. (1985). Social psychological aspects of environmental psychology. In G. Lindzey \& E. Aronson (Eds.), The handbook of social psychology (3rd ed., Vol. 2). Reading, MA: Addison Wesley.

Pol, E. (1988). La psicología ambiental en Europa. Análisis sociohistórico. Barcelona: Anthropos.

Sime, J. D. (1999). What is environmental psychology? Texts, content and context. Journal of Environmental Psychology, 19, 191-206.

Stokols, D. (1978). Environmental psychology. Annual Review of Psychology, 29, 253295.

Stokols, D. (1995). The paradox of environmental psychology. American Psychologist, 50, 821-837.

Stokols, D., \& Altman, I. (Eds.). (1987). Handbook of environmental psychology. New York: John Wiley \& Sons.

Recebido em 5.04.2004

Revisto e encaminhado em 23.02.2005

Aceito em: 7.03.2005 